\title{
Efecto de tres tipos de presas vivas en la larvicultura de bagre blanco (Sorubim cuspicaudus)
}

\author{
Efect of three types of live preys on larviculture of the trans- \\ andean shovelnose catfish (Sorubim cuspicaudus)
}

\author{
Martha Prieto-Guevara, ${ }^{1 *}$ Ph.D, Jhiry Hernández B,${ }^{2}$ Prof. Acuicul, Catalino Gómez R, ${ }^{2}$ Prof. \\ Acuicul, Sandra Pardo C, ${ }^{3}$ Ph.D, Victor Atencio-Garcia, ${ }^{1}$ M.Sc, Priscila V Rosa, ${ }^{4}$ Ph.D.
}

\begin{abstract}
${ }^{1}$ Universidad de Córdoba, Facultad de Medicina Veterinaria y Zootecnia, Departamento de Ciencias Acuícolas, Grupo del Centro de Investigación Piscícola CINPIC. Montería-Córdoba, Colombia. ${ }^{2}$ Universidad de Córdoba, Facultad de Medicina Veterinaria y Zootecnia, Departamento de Ciencias Acuícolas. Montería-Córdoba, Colombia. ${ }^{3}$ Universidad Nacional de Colombia Sede Medellín, Facultad de Ciencias Agrarias, Departamento Producción Animal, Medellín, Colombia. ${ }^{4}$ Universidade Federal de Lavras, Departamento de Zootecnia. Lavras, MG, Brasil. *Correspondencia: alimvi@hotmail.com
\end{abstract}

Recibido: Julio de 2012; Aceptado: Febrero de 2013.

\section{RESUMEN}

Objetivo. Evaluar el efecto de diferentes presas vivas en la larvicultura de bagre blanco (Sorubim cuspicaudus). Materiales y métodos. Al inicio de la alimentación exógena de Sorubim cuspicaudus, se ofreció zooplancton producido en mesocosmos (T1), zooplancton silvestre (T2) y nauplios de Artemia (T3), en concentración de $10.000 \mathrm{zoop} / \mathrm{L}$, dos veces al día, durante seis días. Se utilizaron 18 acuarios de cinco litros de volumen útil, con densidad de 25 Larvas/L, seis réplicas por tratamiento en un diseño al azar. Se estimaron la ganancia en peso (Gp) y longitud (Gl), tasa de crecimiento específico $(G)$, sobrevivencia $(S)$, resistencia al estrés $(R e)$, mortalidad acumulada (Ma) y mortalidad por canibalismo (Mc). Resultados. Las larvas alimentadas con mesocosmos presentaron la mayor sobrevivencia $(81.3 \pm 15.9 \%)$; aunque el mejor crecimiento lo presentaron las larvas alimentadas con zooplancton silvestre (T2) las cuales presentaron la mayor mortalidad $(42.0 \pm 10.7 \%)$ y la menor resistencia al estrés $(30.0 \pm 33.0 \%)$. El canibalismo se observó en todos los tratamientos, oscilando entre 4.0 (T2) y $14.3 \%$ (T1) sin diferencias significativas entre estos valores ( $p>0.05)$. Conclusiones. El uso de zooplancton producido bajo condiciones controladas permitió una alta sobrevivencia, adecuado desempeño y resistencia de las larvas, perfilándose como alternativa viable en la primera alimentación de bagre blanco.

Palabras clave: Alimento vivo, Artemia, larva, mesocosmos (Fuentes: ASFA, CAB). 


\section{ABSTRACT}

Objective. Assess the effect of different live prey in the larviculture of Trans-andean shovelnose catfish (Sorubim cuspicaudus). Materials and methods. At the beginning of exogenous feeding, zooplankton produced in mesocosm (T1), wild zooplankton (T2) and brine shrimp (T3), in concentrations of 10.000 zoop/L, were fed twice daily, for six days. 18 five-liter useful volume aquariums were used, with density of 25 Larvas/L, and six replicas per treatment in a random design. The increase in weight (Gp) length $(\mathrm{Gl})$, the specific growth rate $(\mathrm{G})$, survival $(\mathrm{S})$, stress resistance $(\mathrm{Re})$, accumulated mortality $(\mathrm{Ma})$ and mortality due to cannibalism (Mc) were measured. Results. Larvae fed with mesocosm presented the highest survival rate $(81.3 \pm 15.9 \%)$, although larvae fed wild zooplankton (T2) had the highest growth $(\mathrm{Gp}, \mathrm{Gl}, \mathrm{G})$, and recorded the highest accumulated mortality $(42.0 \pm 10.7 \%)$ and the lowest resistance to stress $(30.0 \pm 33.0 \%)$. Cannibalism was observed in all treatments, ranging from 4.0 (T2) to $14.3 \%$ (T1) no significant difference between these values $(p>0.05)$ was observed. Conclusions. The use of zooplankton produced under controlled conditions allows high survival, adequate performance and resistance of the larvae, emerging as viable alternative for the first feeding of white catfish.

Keywords: Live food, Artemia, mesocosms, larvae (Sources: ASFA, CAB).

\section{INTRODUCCIÓN}

Las larvas de bagre blanco (Sorubim cuspicaudus) se ubican en la categoría de altriciales por presentar poca reserva vitelina y tracto digestivo indiferenciado al inicio de la alimentación exógena; por tal razón, es indispensable el suministro de alimentos vivos (1). Una limitante de mayor importancia en larvicultura de peces es la primera alimentación, registrando mayores índices de mortalidad, principalmente cuando las reservas vitelinas se agotan y los organismos empiezan a ingerir alimento exógeno; este momento se considera crítico; por lo que requieren en esta etapa de su desarrollo una dieta a base de alimentos vivos, fácilmente digeribles y de alto valor nutritivo $(1,2)$. El uso de estos organismos mejora la sobrevivencia y crecimiento de las larvas, las cuales no podrían aprovechar los nutrientes presentes en las dietas inertes debido a que en esta etapa el tracto digestivo no está totalmente desarrollado y no poseen todas las enzimas requeridas para una adecuada digestión (3).

El bagre blanco, como otros pimelódidos, responde bien a las técnicas de reproducción artificial con extracto pituitario de carpa (EPC) y análogos de Salmón (sGnrH-a) $(4,5)$; no obstante falta por definir aspectos que permitan realizar una larvicultura con resultados de crecimiento y sobrevivencia confiables para desarrollar exitosamente tecnologías de producción de alevinos. Un estudio previo reportó que el régimen alimentario del bagre blanco en su fase de alevinaje en estanques en tierra, mostró preferencia por cladóceros (Diaphanosoma, Moinodaphnia, Moina) y copépodos (Argyrodiaptomus, Termocyclops, Mesocyclops) (1); lo cual sugiere que son alimentos que permiten buen desarrollo y crecimiento en sus primeras fases de vida.
La producción de presas específicas en sistema de mesocosmos presenta características importantes para el manejo de la primera alimentación de larvas altriciales, permite ofrecer en condiciones controladas una variedad de presas y tamaños, que sirven de estímulo al comportamiento predador de la larva, se evitan influencias ambientales desfavorables y aumenta la tasa de supervivencia debido a la ausencia de predadores y patógenos que coexisten con el zooplancton silvestre (2). Por otro lado, la estructura y composición del zooplancton silvestre como alimento, puede variar a lo largo del año, pudiendo influir sobre el desempeño de la larvicultura $(1,6)$. Entonces, con el suministro de zooplancton seleccionado se puede contribuir a mejorar el proceso productivo de la larvicultura e incrementar la oferta de alevinos para el fomento del cultivo de una especie.

De este modo, el sistema de mesocosmos surge como alternativa en el manejo de la primera alimentación de peces tropicales, paralelo a sistemas tradicionales como la alimentación con zooplancton silvestre y el suministro de nauplios de Artemia. El presente estudio evaluó la larvicultura de bagre blanco al inicio de la alimentación exógena, alimentando con zooplancton silvestre, nauplios de Artemia y zooplancton generado en sistemas de mesocosmos para determinar su incidencia en la sobrevivencia, crecimiento y calidad de la larva.

\section{MATERIALES Y MÉTODOS}

Material biológico y unidades experimentales. El estudio se realizó en el Centro de Investigación Piscícola de la Universidad de Córdoba CINPIC (Montería, Córdoba, Colombia), cuyas coordenadas geográficas son $8^{\circ} 48^{\prime}$ de Latitud 
Norte y $75^{\circ} 22^{\prime}$ de Longitud Oeste, con altura de $15 \mathrm{msnm}$ y valores anuales promedios de temperatura de $27.5^{\circ} \mathrm{C}$, humedad relativa del $85 \%$ y precipitaciones de $1100 \mathrm{~mm}$. Se utilizaron larvas obtenidas mediante reproducción artificial, de reproductores de bagre blanco mantenidos en cautiverio, inducidos con Ovaprim ${ }^{\circledR}$ en dosis única de $0.3 \mathrm{ml} / \mathrm{Kg}$ de peso, en la base de la aleta pectoral, tanto para machos como para hembras $(4,5)$, mantenidos en piletas de $6 \mathrm{~m}^{3}$; a partir de diez horas post-inducción se revisó cada media hora para determinar el momento de la ovulación. La fertilización se realizó en seco, posteriormente estos fueron transferidos a incubadoras cilindro-cónicas de $60 \mathrm{~L}$ de capacidad y flujo ascendente continuo (4-6 I/ $\mathrm{min})$.

Las larvas recién eclosionadas fueron transferidas a incubadoras con volumen de 200 litros y mantenidas allí hasta las 40 horas post-eclosión (HPE). Todas las larvas procedían del desove de una sola hembra, para evitar el sesgo en la calidad de las larvas, fueron contadas individualmente y dispuestas a densidad de 25 larvas/l; para un total de 125 larvas en cada una de las 18 unidades experimentales, conformadas por acuarios de vidrio con volumen operativo de 5 litros, con tres de sus lados cubiertos con plástico negro para aislar visualmente los acuarios, reducir la incidencia de la luz y minimizar los factores de estrés en los organismos. Al inicio de la alimentación exógena se realizó una descripción general de larvas, considerando las características de los ojos, boca, saco vitelino, barbicelos y pigmentación. Adicionalmente se determinó la abertura bucal máxima, mediante la ecuación:

$A b m=L m s * \sqrt{2}$

(Lms, longitud del maxilar superior).

Se utilizó agua del río Sinú, previamente filtrada por un filtro lento de arena, tratada con sulfato de aluminio $(20 \mathrm{mg} / \mathrm{l})$, aireada y reposada durante tres días para obtener apropiada calidad. La aireación fue individual en cada acuario, diariamente se registró la temperatura, oxígeno disuelto con oxímetro digital (YSI, 550A, USA) y $\mathrm{pH}$ con pH-metro digital (WTW, 320, Alemania). La dureza total, alcalinidad y amonio total fueron medidos al inicio, en la mitad y al final del estudio. La alcalinidad fue determinada por el método de la fenolftaleína, utilizando reactivos $\mathrm{HACH}$ (ácido sulfúrico, fenolftaleína y verde de bromocresol), con un titulador digital $(\mathrm{HACH}$, USA). La dureza se registró con el método de titulación EDTA con sodio a concentración de $0.08 \mathrm{M}$ mediante la utilización de un titulador digital (HACH, USA). El amonio total se determinó mediante el método de titulación ácida utilizando reactivos $\mathrm{HACH}$ (agua desmineralizada, acido sulfúrico $8 \mathrm{~N}$ y solución indicadora $\mathrm{HACH}$, USA). La mortalidad diaria y total se determinó colectando las larvas muertas por acuario antes y después de la alimentación. Heces y alimento no consumido fueron retirados mediante sifoneo dos horas después de la alimentación y seguidamente se realizó recambio hasta de $30 \%$ del volumen de agua total.

Se suministró dos raciones diarias (8:00 am y 5:00 pm), durante seis días, de los siguientes alimentos: T1, zooplancton entre $250-400 \mu \mathrm{m}$ producidos en mesocosmos compuesto por copépodos del género Diaptomus sp (41\%) y cladóceros (59\%) de los géneros Diaphanosoma sp, Moina sp y Moinodaphnia sp; T2, zooplancton silvestre 250$400 \mu \mathrm{m}$ compuesto por copépodo cyclopoides (52\%) y cladóceros $(48 \%) ;$ T3, nauplios recién eclosionados de Artemia (250 $\mu \mathrm{m})$; para un total de 50000 presas en cada acuario por día (densidad $10 \mathrm{ind} / \mathrm{ml}$ ). La densidad del zooplancton se determinó volumétricamente contando tres alícuotas de $1 \mathrm{ml}$ en cámara multicelda con ayuda de estereoscopio (Leica, Wild MZ8, Alemania), se determinó el número total de organismos por mililitro y la composición porcentual de cada grupo por tratamiento.

Producción de zooplancton. Cepas del copépodo calanoide Diaptomus sp y los cladóceros Diaphanosoma sp, Moina sp., y Moinodaphnia sp., obtenidas en el Laboratorio de Alimento Vivo de la Universidad de Córdoba; fueron mantenidas en frascos de $250 \mathrm{ml}$, usando como alimento microalgas Ankistrodesmus y Scenedesmus en concentración de $1 \times 10^{5} \mathrm{cel} / \mathrm{ml}$ y $3 \times 10^{5} \mathrm{cel} / \mathrm{ml}$ para copépodos y cladóceros, respectivamente; de acuerdo con método implementado por Martínez et al (7). Se realizó proliferación de biomasas en frascos de $3 \mathrm{I}$, con aireación moderada, iluminación constante y renovación de $30 \%$ del volumen de agua cada tres días, mediante el uso de filtros de $40 \mathrm{~mm}$ para retener cladóceros y nauplios de copépodos. El incremento de biomasa en el tiempo se realizó siguiendo el método descrito por Prieto et al (8), el cual consiste básicamente en realizar inóculos sucesivos, empleando el método de serial de volúmenes.

Para la producción de zooplancton en el sistema mesocosmos se utilizó agua filtrada a $40 \mathrm{~mm}$, en un sistema modular en concreto de $6 \mathrm{~m}^{3}$ en exterior, protegido con techo traslucido, aireación constante, fotoperiodo natural y temperatura ambiente $\left(28 \pm 1^{\circ} \mathrm{C}\right)$. Para conformar el mesocosmos se inocularon biomasas de copépodos y cladóceros en relación 65:35 respectivamente; producidas con aireación individualmente en tanques circulares en fibra de vidrio (capacidad $1000 \mathrm{I}$ ); acorde a la 
diferencia en la estrategia reproductiva y método establecido por Martínez et al (7). Una vez en producción, se realizó cosecha parcial diaria del cultivo de mesocosmos con red planctónica de $100 \mu \mathrm{m}$ y selección a través de tamices entre 250 y $400 \mu \mathrm{m}$ para la alimentación de las larvas en estudio. Luego de establecer la concentración de zooplancton, se calculó la ración diaria para cada unidad experimental por el método volumétrico, ajustando la densidad final descrita.

Zooplancton silvestre y artemia. Mediante arrastres diarios en estanques en tierra fertilizados, utilizando red planctónica de $35 \mu \mathrm{m}$ y sistema de tamices de tambor, se colectó y separó entre 250 y $400 \mu \mathrm{m}$ zooplancton silvestre. Previo al suministro, se contó e identificó los grupos principales (Rotíferos, Cladóceros y Copépodos). Los náuplios Instar I de Artemia fueron obtenidos mediante eclosión diaria de cistos, en incubadoras cilíndricas conteniendo agua de mar a $35 \%$ de salinidad y $30^{\circ} \mathrm{C}$, con aireación fuerte e iluminación directa constante mediante lámparas fluorescentes con 2000 lux en la superficie del agua. La cosecha se realizó después de 24 horas, para lo cual se retiró la aireación y se aprovechó el fototropismo positivo de los nauplios para que se concentraran en el fondo de las incubadoras.

Parámetros de desempeño. Al inicio del estudio se tomaron 100 larvas y entre 15 a 20 larvas por cada acuario al final del experimento, se preservaron en formalina al 4\% (v/v) tamponada; para su posterior evaluación. Las larvas fueron pesadas en balanza analítica $0.01 \mathrm{mg}$ (Ohaus Adventurer, Dinamarca) y medidas desde la punta del hocico hasta el final de la aleta caudal, con la ayuda de un analizador de imagen (Axiovision 4.3, Alemania) y estereoscopio (Leica, Wild MZ8, Alemania) con cámara digital incorporada (Canon, Power G10, Japón).

La ganancia en peso (Gp) y la ganancia en longitud (Gl) se estimó empleando las ecuaciones: Gp $(\mathrm{mg})=$ Pmf - Pmi; donde Pmf y Pmi corresponden al peso medio final e inicial respectivamente. Gl $(\mathrm{mm})=\mathrm{Ltf}-\mathrm{Lti}$; donde Ltf y Lti corresponden a la longitud total final e inicial respectivamente. La tasa de crecimiento específico (G) se estimó con la fórmula: G (\%/día)= Ln (Pmf-Pmi)/t x 100; donde Pmf y Pmi corresponden al peso final e inicial respectivamente, t es el tiempo de cultivo expresado en días y Ln es el logaritmo neperiano.

Al final del experimento se contaron las larvas vivas y la sobrevivencia final se calculó con la fórmula: $\mathrm{S}(\%)=$ (número final de larvas/número inicial de larvas) x 100. Igualmente se retiraron 20 larvas de cada unidad experimental con una nasa con ojo de malla de $400 \mu \mathrm{m}$, se colocaron fuera del agua, sobre papel absorbente durante ocho minutos; posteriormente se transfirieron a un recipiente con agua de la misma unidad experimental; 20 minutos más tarde se contaron las larvas que sobrevivieron y de esta manera se calculó la sobrevivencia a la prueba de resistencia al estrés; este método fue utilizado por Kerguelén-Durango et al (6) en Prochilodus magdalenae como un indicador de calidad larval. Diariamente, se colectó y contó por cada unidad experimental las larvas muertas.

La mortalidad diaria (Md) se calculó con la siguiente fórmula: Md $(\%)=$ (número de larvas muertas día/número inicial de larvas) x 100 . Para establecer la mortalidad acumulada (Ma), se cuantificó el número total de larvas muertas por día y se utilizó la ecuación: $\mathrm{Ma}$ (\%)=(número total de larvas muertas/número inicial de larvas) x 100. Se consideró como mortalidad por canibalismo (Mc) a la diferencia entre la mortalidad total y la mortalidad acumulada, esta mortalidad se estimó mediante la fórmula: $\mathrm{Mc}(\%)=($ (número inicial Larvas - número final Larvas) - mortalidad acumulada $) /\left(\mathrm{N}^{\circ}\right.$ inicial Larvas) $\times 100$.

Análisis estadístico. Se utilizó un diseño completamente aleatorio con tres tratamientos, cada uno con seis repeticiones. A todas las variables se les realizó las pruebas de normalidad (ShapiroWilk) y homogeneidad de varianza (KolmogorovSmirnov), previamente transformadas mediante la función arcoseno. Luego se analizaron mediante análisis de varianza (ANOVA) y cuando se observaron diferencias estadísticas significativas se aplicó la prueba de Rango Múltiple de Diferencia Mínima Significante (LSD), utilizando para todos los casos una significancia de $p<0.05$. El análisis estadístico fue realizado con ayuda del programa SAS (9).

\section{RESULTADOS}

En la tabla 1 se presentan los valores promedios de temperatura, oxígeno disuelto, $\mathrm{pH}$, alcalinidad, dureza y amonio total en el agua utilizada durante el experimento en los diferentes tratamientos. Ninguno de los parámetros analizados presentó diferencias significativas ( $p>0.05)$.

Tabla 1. Calidad del agua en la larvicultura de bagre blanco alimentadas con tres tipos de organismos zooplanctónicos durante seis días.

\begin{tabular}{lccc}
\hline & T1 & T2 & T3 \\
\hline Temperatura $\left({ }^{\circ} \mathrm{C}\right)$ & $27.8 \pm 0.7^{\mathrm{a}}$ & $28.0 \pm 1.2^{\mathrm{a}}$ & $27.7 \pm 0.7^{\mathrm{a}}$ \\
Oxígeno disuelto $(\mathrm{mg} / \mathrm{l})$ & $6.6 \pm 0.4^{\mathrm{a}}$ & $6.5 \pm 0.6^{\mathrm{a}}$ & $6.2 \pm 0.7^{\mathrm{a}}$ \\
$\mathrm{pH}$ & $7.7 \pm 0.3^{\mathrm{a}}$ & $7.4 \pm 0.5^{\mathrm{a}}$ & $7.4 \pm 0.5^{\mathrm{a}}$ \\
Alcalinidad $(\mathrm{mg} / \mathrm{l})$ & $72.5 \pm 9.4^{\mathrm{a}}$ & $71.2 \pm 9.2^{\mathrm{a}}$ & $75.8 \pm 12.1^{\mathrm{a}}$ \\
Dureza total $(\mathrm{mg} / \mathrm{l})$ & $68.1 \pm 6.9^{\mathrm{a}}$ & $64.8 \pm 5.0^{\mathrm{a}}$ & $64.1 \pm 3.5^{\mathrm{a}}$ \\
Amonio total $(\mathrm{mg} / \mathrm{l})$ & $0.06 \pm 0.03^{\mathrm{a}}$ & $0.08 \pm 0.02^{\mathrm{a}}$ & $0.07 \pm 0.01^{\mathrm{a}}$ \\
\hline
\end{tabular}


Las larvas de bagre blanco iniciaron la primera alimentación entre 48 y $51 \mathrm{HPE}$, a temperatura promedio del agua de $28.5 \pm 0.7^{\circ} \mathrm{C}$, con peso promedio de $1.0 \pm 0.0 \mathrm{mg}$, longitud total promedio de $5.6 \pm 0.4 \mathrm{~mm}$ y abertura bucal inicial promedio de $622.3 \pm 70.2 \mu \mathrm{m}$. El cuerpo se observó traslúcido, con presencia del tubo digestivo formado, sin diferenciación de glándulas anexas, los barbillones blanquecinos en formación y lámelas branquiales definidas, boca en posición terminal y ojos completamente pigmentados.

En la tabla 2 se registró el desempeño en crecimiento de las larvas de bagre blanco. Las larvas alimentadas con zooplancton silvestre (T2) presentaron la mayor Gp (24.9 2.2


estadísticamente a los otros tratamientos $(\mathrm{p}<0.05)$; también la mayor Gl fue también fue registrada en las larvas alimentadas con T1 $(10.5 \pm 0.7 \mathrm{~mm})$, pero sin observarse diferencia significativa $(p>0.05)$ con las alimentadas con zooplancton producido en sistema de mesocosmos (T2, 11.3 $\pm 0.5 \mathrm{~mm})$.

Tabla 2. Valores promedio de ganancia en peso (Gp), en longitud (GI) y tasa de crecimiento específico (G) de larvas de bagre blanco alimentadas con tres tipos de organismos zooplanctónicos durante seis días.

\begin{tabular}{cccc}
\hline Tratamiento & $\mathbf{G P}(\mathbf{m g})$ & $\mathbf{G l}(\mathbf{m m})$ & $\mathbf{G}(\% /$ día $)$ \\
\hline T1 & $19.7 \pm 1.4^{\mathrm{b}}$ & $10.5 \pm 0.7^{\mathrm{a}}$ & $50.5 \pm 1.1^{\mathrm{b}}$ \\
T2 & $24.9 \pm 2.2^{\mathrm{a}}$ & $11.3 \pm 0.5^{\mathrm{a}}$ & $54.4 \pm 1.3^{\mathrm{a}}$ \\
T3 & $19.4 \pm 2.9^{\mathrm{b}}$ & $9.6 \pm 0.3^{\mathrm{b}}$ & $50.1 \pm 2.4^{\mathrm{b}}$ \\
\hline
\end{tabular}

Letras diferentes, en la misma columna, indican diferencias significativas $(p<0.05)$.

En la tabla 3 se registran los valores promedios de sobrevivencia al final del ensayo (S) y la sobrevivencia después que las larvas fueron sometidas a la prueba de resistencia al estrés (Re). La sobrevivencia final osciló entre $54.0 \pm 14.1 \%$ (T2) y $81.3 \pm 15.9 \%$ (T1) sin observarse diferencia significativa entre los diferentes tratamientos $(p>0.05)$. La mayor sobrevivencia a la prueba de resistencia al estrés se observó en larvas alimentadas con Artemia (95.6 $\pm 5.4 \%)(p<0.05)$;

Tabla 3. Valores promedios de sobrevivencia (S) y prueba de resistencia al estrés (Re) de larvas de bagre blanco alimentadas con tres tipos de organismos zooplanctónicos durante seis días.

\begin{tabular}{ccc}
\hline Tratamiento & S (\%) & Re (\%) \\
\hline T1 & $81.3 \pm 15.9^{\mathrm{a}}$ & $61.3 \pm 40.9^{\mathrm{b}}$ \\
T2 & $54.0 \pm 14.1^{\mathrm{a}}$ & $30.0 \pm 33.0^{\mathrm{b}}$ \\
T3 & $64.8 \pm 16.3^{\mathrm{a}}$ & $95.6 \pm 5.4^{\mathrm{a}}$ \\
\hline
\end{tabular}

Letras diferentes, en la misma columna, indican diferencias significativas $(p<0.05)$. seguidas por las alimentadas con zooplancton producido en sistema mesocosmo (61.3 $440.9 \%)$ y silvestre $(30.0 \pm 33.0 \%)$ sin presentarse diferencias significativas entre estos valores.

En el primer día, la mortalidad diaria osciló entre $0.5 \pm 0.7 \%$ (T1) y $6.8 \pm 8.5 \%$ (T2) sin observarse diferencia estadística entre estos valores ( $p>0.05)$; entre los días 2 y 4 de la larvicultura fue mayor en las larvas alimentadas con zooplancton silvestre (T2) registrando mortalidades diarias por encima de $9 \%$; mientras que los días 5 y 6 la mortalidad diaria fue mayor en las larvas alimentadas con nauplios de Artemia (T3), registrando mortalidades diarias cercanas a $6 \%(p<0.05)$. Las larvas alimentadas con zooplancton producido en sistema de mesocosmo (T1) registraron la menor mortalidad diaria durante el ensayo, en ninguno de los días superó en promedio $2.0 \%$ (Figura 1 ).

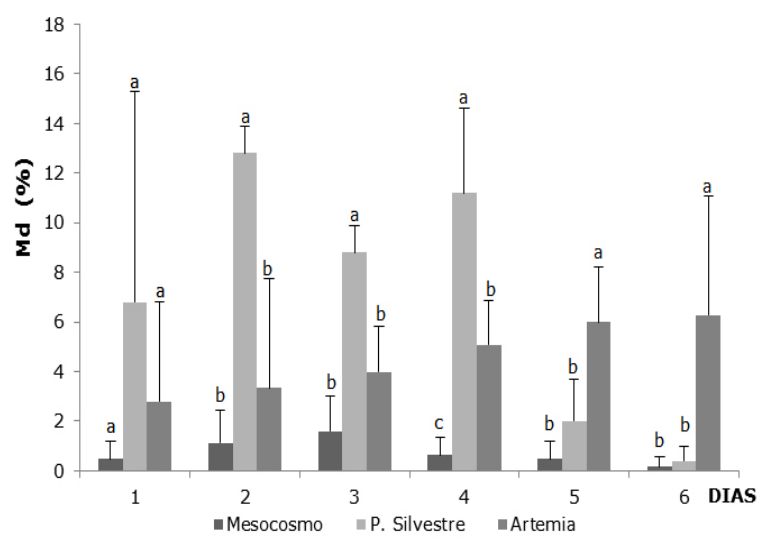

Figura 1. Valores promedios de mortalidad diaria (Md) de larvas de bagre blanco alimentadas con tres tipos de organismos zooplanctónicos durante seis días. Letras diferentes indican diferencias significativas $(p<0.05)$.

En la tabla 4 se muestra la mortalidad acumulada (Ma) al final del ensayo y la mortalidad por causa del canibalismo. La mayor mortalidad acumulada se obtuvo en las larvas alimentadas

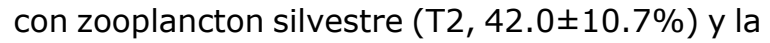
menor en las larvas alimentadas con mesocosmos $(\mathrm{T} 1,4.5 \pm 2.7 \%)$ observándose diferencia significativa entre los tratamientos $(p<0.05)$. Diariamente se observó canibalismo, al momento de retirar las larvas muertas se encontraban larvas mordidas, restos como cabezas y colas; al final del experimento algunas larvas habían desaparecido por completo. El canibalismo osciló entre $4.0 \pm 3.4 \%$ (T2) $14.2 \pm 17.2 \%$ (T3) $\sin$ observarse diferencia significativa entre estos valores promedios $(p>0.05)$. 
Tabla 4. Mortalidad acumulada (Ma) y mortalidad por canibalismo (Mc) en la larvicultura de bagre blanco alimentadas con tres tipos de organismos zooplanctónicos durante seis días.

\begin{tabular}{ccc}
\hline Tratamiento & Ma & Mc \\
\hline $\mathrm{T} 1$ & $4.5 \pm 2.7^{\mathrm{c}}$ & $14.2 \pm 17.2^{\mathrm{a}}$ \\
$\mathrm{T} 2$ & $42.0 \pm 10.7^{\mathrm{a}}$ & $4.0 \pm 3.4^{\mathrm{a}}$ \\
$\mathrm{T} 3$ & $27.3 \pm 8.2^{\mathrm{b}}$ & $7.9 \pm 9.0^{\mathrm{a}}$ \\
\hline
\end{tabular}

Letras diferentes, en la misma columna, indican diferencias significativas $(p<0.05)$.

\section{DISCUSION}

La calidad del agua utilizada en el ensayo está dentro del rango adecuado para la larvicultura de bagre blanco según lo reportado por Espinosa (4). Por tanto la calidad del agua utilizada en este estudio, no afectó el crecimiento ni la sobrevivencia, ya que se mantuvieron en los rangos adecuados para el cultivo y larvicultura de esta especie.

En larvicultura, el momento de la alimentación exógena es determinante pues en esta etapa, se presentan las mayores tasas de mortalidad debido a que las larvas deben aprender y acostumbrarse a capturar y a consumir alimento (1). El inicio de la alimentación exógena de bagre blanco registrada en este estudio (48-51 HPE, temperatura promedio de $27.5^{\circ} \mathrm{C}$ ); es similar al rango de tiempo reportado, en la misma franja de temperatura, para otros pimelódidos como bagre rayado Pseudoplatystoma fasciatum y yaque Leairius marmaratus $(10,11)$. La abertura bucal de bagre blanco al inicio de la alimentación exógena registrada en el presente estudio $(622 \pm 70.2 \mu \mathrm{m})$; se puede considerar en el rango de la abertura bucal reportada para otros pimelódidos como Leiarius marmoratus $(532.7 \pm 7.8 \mu \mathrm{m})$ por Ramirez-Merlano et al (11).

El conocimiento sobre la dimensión de la abertura bucal es fundamental para tener una idea acerca del tamaño del alimento que debe suministrarse a las larvas; ya que estas seleccionan el alimento teniendo en cuenta el tamaño de la presa $(1,12)$. Considerando la abertura bucal de bagre blanco al inicio de la alimentación exógena, es posible inferir que todas las presas tenían el tamaño adecuado para manejo de primera alimentación; el tamaño del zooplancton silvestre utilizado $(250-400 \mu \mathrm{m})$ fue adecuado y de fácil captura para las larvas. En este rango de tamaño la mayoría de los copépodos se encontraban en estadio de náuplios y copepodito; siendo considerados como alimentos adecuados para las larvas, ya que a pesar de ser partículas de mayor tamaño presentan un movimiento más lento permitiendo a los peces una fácil captura (13). Atencio-García et al (12), encontraron que en el manejo de la primera alimentación de yamú Brycon amazonicus el consumo de zooplancton de mayor tamaño permite mejores tasas de crecimiento.

El mayor incremento en $\mathrm{Gl}, \mathrm{Gp}$ y $\mathrm{G}$ se registró en las larvas de bagre blanco alimentadas con zooplancton silvestre. A pesar de ser valores mayores con respecto a los otros tratamientos (mesocosmos y Artemia), no representan una perspectiva real del desempeño de la especie por efecto del tratamiento en particular, sino un efecto de la alta mortalidad (42\%) que conllevó a una baja densidad, lo cual permitió a las larvas un mejor aprovechamiento del alimento suministrado, por existir menor competencia por este y mayor espacio disponible.

Las larvas alimentadas con mesocosmos, exhibieron valores aceptables de $\mathrm{Gl}, \mathrm{Gp}$ y $\mathrm{G}$ registrando una alta sobrevivencia $(81.3 \pm 15.9 \%)$ y baja mortalidad $(4.48 \%)$. Teniendo en cuenta estas circunstancias, los registros de crecimiento y sobrevivencia perfilan el mesocosmos como una alternativa eficiente de alimentación para la larvicultura de bagre blanco. Los menores resultados de crecimiento se encontraron cuando se alimentó con nauplios de Artemia, que a pesar de registrar sobrevivencias aceptables $(64.8 \pm 16.3 \%$ ) y crecimiento (Gl y $\mathrm{Gp}$ ), el desempeño fue inferior al registrado con mesocosmos, no obstante que se exigía menor competencia de las larvas por el espacio y la alimentación.

Estos resultados señalan una de las grandes ventajas que tienen los organismos zooplanctónicos cultivados en mesocosmos sobre la Artemia, como es el hecho de pertenecer al mismo hábitat de las larvas objeto de cultivo (agua dulce), donde se pueden mantener en buenas condiciones y por más tiempo suspendidos en el agua, permitiéndole a la larva un mejor aprovechamiento del alimento, a diferencia de los nauplios de Artemia que por ser de otro hábitat (agua salada), después de un período de tiempo corto, los que no son consumidos, van al fondo y no son aprovechados por las larvas en cultivo.

En cuanto a la sobrevivencia, el alto valor $(81.3 \pm 15.9 \%)$ registrado en el presente estudio en larvas alimentadas a base de cultivos mixtos de copépodos y cladóceros en sistema de mesocosmos, permite sugerir que estos organismos zooplanctónicos presentaron las características requeridas por las larvas, tales como homogeneidad en la diversidad de especies y apropiada calidad nutricional. Estos alimentos permitieron una adecuada digestión y aprovechamiento de los nutrientes, al ser cultivados y monitoreados en condiciones controladas. Además, al ser seleccionados por tamaño se permitió su ingesta obteniéndose así buenos resultados de crecimiento en longitud, peso, tasa de crecimiento especifico 
y un ítem alimentario que se convierte en una alternativa viable como alimento para manejar la primera alimentación de bagre blanco. Otros estudios también han reportado buenas sobrevivencia cuando se utiliza zooplancton seleccionado y cultivado como lo reportado por Marciales-Caro et al (13) quienes obtuvieron sobrevivencia de $63.1 \%$ alimentados con cladóceros (Moina sp y Diaphanosoma $s p$ ) a razón de 0.5 ind/ml en la primera alimentación de larvas de Pseudoplatystoma sp.

A diferencia, el zooplancton silvestre no cuenta con estas características, ya que la estructura y composición (especies y nutrientes) del mismo puede variar con la época del año, influyendo así sobre el desempeño de las larvas, al mismo tiempo es frecuente la presencia de predadores de larvas entre sus componentes $(1,6,12)$; además este tipo de predación se puede sugerir como responsable de la alta mortalidad y elevada variabilidad (Coef de variación $=125 \%$ ) el primer día en este tratamiento. Por su parte, el uso de náuplios de Artemia en larvicultura, además de ofrecer resultados inferiores en sobrevivencia $(64.8 \pm 16.3 \%)$ y crecimiento en longitud, peso y tasa de crecimiento específico, resulta oneroso en la larvicultura del bagre blanco. Según Díaz-Olarte et al (14), los costos de producción de la Artemia, como presa para el manejo de primera alimentación; es una de las causas de búsqueda de nuevas alternativas de alimentación en la larvicultura, como el uso de especies del zooplancton.

Nuñez et al (15), reportaron sobrevivencia en larvas de bagre rayado Pseudoplatustoma magdaleniatum de $55.0 \pm 13.2 \%$ alimentados con Artemia, siendo estos valores inferiores a los registros en este estudio (64.8 $\pm 16.3 \%$ ). En cuanto al zooplancton silvestre, estos autores reportan sobrevivencia de $44.0 \pm 14.8 \%$; mientras que en este trabajo, la sobrevivencia, con este mismo tratamiento, fue

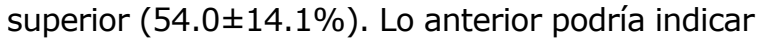
que la estructura y composición del zooplancton silvestre varía en el tiempo y por tanto, también los resultados en su uso como presa viva en larvicultura de peces tropicales.

Adicionalmente el zooplancton silvestre, presenta otros inconvenientes cuando es utilizado como alimento para larvas, ya que al ser extraído del medio natural tiene el riesgo de contener organismos predadores, dentro de los cuales se destacan los copépodos ciclopoides, muy abundantes en las aguas continentales del neotrópico (16). Estos resultados permiten sugerir lo inadecuado que resulta el manejo de la primera alimentación de bagre blanco con zooplancton silvestre por los riesgos de introducir copépodos predadores que afectan el desempeño de la larvicultura, principalmente la sobrevivencia. Al mismo tiempo, la constitución y perfil nutricional del zooplancton natural, varía con relación a la temporada y el nivel de nutrientes presentes en el cuerpo de agua (1) lo cual no garantiza la composición estable del zooplancton en el tiempo, al momento de realizar la larvicultura.

La prueba de resistencia al estrés permite estimar la calidad y viabilidad de las larvas acorde al tipo de alimento empleado, así, una mayor sobrevivencia a esta prueba indica una mejor condición de la larva y por tanto una mejor calidad nutricional del alimento ofrecido en asocio con las condiciones de manejo $(1,6)$.

Las larvas alimentadas con Artemia presentaron los valores más altos en la resistencia al estrés $(95 \%)$, con relación al tratamiento con mesocosmos (61\%). Esto es una ventaja nutricional de la alimentación a base de Artemia, permitiendo la producción de una larva resistente a la manipulación y traslado al sitio de cultivo (estanques de alevinajes). Aún así, los resultados obtenidos en el tratamiento con mesocosmos, también son adecuados, lo que indica que este alimento ofrece características nutricionales que permiten a las larvas alta resistencia para el manejo y transporte. En contraste, las larvas alimentadas con zooplancton silvestre presentaron resistencia reducida al estrés $(30.0 \pm 33.0 \%)$, evidenciando baja condición para su manejo, lo cual no garantiza resistencia para el empaque, transporte y cambios ambientales a que es sometida la larva para continuar con su cultivo (alevinaje).

Valores similares a los obtenidos con nauplios de Artemia en este estudio fueron reportados por Nuñez et al (15) en larvicultura de bagre rayado, alimentados con Artemia (91.0\%). Estos resultados sugieren que Artemia sigue siendo un alimento de muy buena calidad nutricional y que larvas alimentadas con mesocosmos se comportan de forma similar a los resultados obtenidos por estos autores, al alimentar con zooplancton de mayor tamaño (250-400 $\mu \mathrm{m})$.

En las larvas alimentadas con mesocosmos, la mortalidad registró el valor más bajo entre los tratamientos evaluados. Esto muestra la calidad del alimento en cuanto a especies seleccionadas para establecer el mesocosmos, donde no sólo se tuvo en cuenta su contenido nutricional sino también la selectividad de la especie por estos zooplánctontes, los patrones de coexistencia de los mismos en el medio de cultivo y la ausencia de predadores. 
En contraste, en las larvas alimentadas con Artemia la mortalidad fue mayor. A pesar de suministrar el alimento en estado de nauplios, los cuales son atractivos por su color y no son agresivos, la mortalidad fue superior al tratamiento con mesocosmos. Este resultado pudo estar relacionado por el poco tiempo de disponibilidad de los nauplios de Artemia en el medio para el consumo por parte de las larvas, lo cual está influenciado por la reducida sobrevivencia de este alimento en el agua dulce. Esto hace que los individuos que no son consumidos por las larvas mueran y lleguen al fondo como ya fue mencionado. La mayor mortalidad se registró en las larvas alimentadas con zooplancton silvestre, sugiriéndose como posibles causas, la presencia de predadores dentro del alimento suministrado.

En general, los resultados de mortalidad evidencian la apropiada calidad del mesocosmo como alimento adecuado para la larvicultura de bagre blanco en su primera alimentación exógena, momento en el cual se registran las mayores mortalidades. Al ser comparado con el zooplancton silvestre, influenciado por su estructura y composición la cual puede variar a lo largo del año, con presencia de copépodos Ciclopoides; predadores que influyen sobre el desempeño de las larvas en cultivo (1, 12). Así, la baja mortalidad asociada a una alta sobrevivencia registrada en larvas alimentadas con mesocosmos, permite avanzar en el manejo de la primera alimentación de bagre blanco sin depender del zooplancton silvestre y sus riesgos asociados, ni de Artemia por su elevado costo.

En otros pimelódidos, Nuñez et al (15), reportaron mortalidades de $46.3 \pm 6.0 \%$ para larvas de bagre rayado alimentadas con náuplios de Artemia, siendo esta muy superior a la registrada en este trabajo con mesocosmos. Estudios anteriores presentan a Artemia como el mejor alimento para esta etapa en larvicultura de peces, en el presente trabajo se evidencia que el mesocosmos la supera en cuanto a sobrevivencia y crecimiento, mostrándose como un alimento más apropiado en la primera alimentación exógena para larvas de Bagre blanco.

El canibalismo es un aspecto relevante en la larvicultura (17). En todos los tratamientos se registró canibalismo y en general fue bajo (4-14.0\%); observándose que cuando la mortalidad por otras causas fue mayor, esta conducta registró menores valores (Tabla 4). Por tanto, el canibalismo en las larvas alimentadas con zooplancton producido en sistema mesocosmos se sugiere relacionada con la elevada sobrevivencia $(81.3 \pm 15.9 \%)$ en ese tratamiento, porque conlleva a menor espacio disponible para las larvas facilitando la probabilidad de encuentro entre las mismas, incitando con esto al canibalismo. En larvas alimentadas con Artemia, se registró $7.9 \%$, algo razonable dada la mayor mortalidad (27.5 $\pm 8.2 \%)$, lo cual proporciona más espacio entre las larvas y menor posibilidad de encuentro. Por consiguiente, en el tratamiento con zooplancton silvestre la mortalidad por canibalismo fue de $4.0 \pm 3.4 \%$, siendo la más baja en el experimento y ratificando que a mayor mortalidad mayor espacio y menor probabilidad de encuentro entre las larvas y con esto menor mortalidad por canibalismo.

Diaz-Olarte et al (14) observaron mayor canibalismo en larvicultura Pseudoplatystoma al incrementar las densidades siembra de 15 Larvas/L a 45 Larvas/L. Nuñez et al (15) reportaron mortalidad por canibalismo de $2.4 \pm 9.6 \%$ en larvas de bagre rayado Pseudoplatystoma sp., alimentadas con Artemia, valor inferior al obtenido en el presente estudio con el tratamiento de mesocosmos, dato también razonable dado que la sobrevivencia $(81.3 \pm 15.9 \%)$ obtenida en este estudio en el tratamiento con mesocosmos es superior a la reportada por estos autores $(53.7 \pm 13.2 \%)$.

En este estudio se evidenció que el tipo de presa tiene efecto directo sobre el desempeño en larvicultura, indicando que entre las presas usadas, el mesocosmos entre 250-400 $\mu \mathrm{m}$ es una alternativa importante como alimento para larvas de bagre blanco; permitiendo una elevada sobrevivencia (superior al $80 \%$ ) y adecuadas longitud, peso y resistencia al estrés de las larvas, siendo similares a los obtenidos con Artemia y contrastando con el bajo desempeño cuando se alimenta con zooplancton silvestre. Acorde a los resultados obtenidos, el día crítico en la fase de manejo de la primera alimentación del Bagre blanco ocurre entre el segundo y tercer día de larvicultura.

En conclusión el uso de zooplancton producido bajo condiciones controladas permitió una alta sobrevivencia, adecuado desempeño y resistencia de las larvas, perfilándose como alternativa viable en la primera alimentación de bagre blanco.

\section{Agradecimientos}

Al Centro de Investigaciones Piscícolas de la Universidad de Córdoba - CINPIC por facilitar sus instalaciones y material biológico para desarrollar este trabajo; a la Oficina de Investigación y Extensión de la Universidad por su apoyo económico. A Yamid Hernández Julio, M.Sc., por su dedicación y apoyo al editar y formatear este manuscrito. 


\section{REFERENCIAS}

1. Prieto $\mathrm{MJ}$, Atencio VJ. Zooplancton y mesocosmo en la larvicultura de peces neotropicales. Rev Bras Zootecn 2008; 37(Supl):1-17.

2. Prieto $M$, Castaño $F$, Sierra J, Logato $P$, Botero J. Alimento vivo en la larvicultura de peces marinos. Copépodos y mesocosmos. Rev MVZ Córdoba 2006a; 11(1):30-36.

3. Prieto MJ, Moraes G, Okamura D, Logato PV, Araujo FG. Tipo de alimento, sobrevivência e desempenho inicial de Pós-larvas de Pacu (Piaractus mesopotamicus). Cienc Agrotec 2006c; 30(5):1002-1007.

4. Espinosa JA. Estandarización de un protocolo para la crioconservación de semen de bagre blanco Sorubim cuspicaudus. [Tesis de Maestría], Montería, Colombia: Universidad de Córdoba, Facultad de Ciencias Básicas, Departamento de Química; 2012.

5. Atencio-García V. Producción de alevinos de especies nativas. Rev MVZ Córdoba 2001; 6(1):9-14.

6. Kerguelén-Durango E, Atencio-García V, Wadnipar L, Narváez A. Manejo de la primera alimentación del bocachico (Prochilodus magdalenae). Rev MVZ Córdoba 2003; 8(1):254-260.

7. Martínez JL, Ortiz ME, Prieto MJ. Estandarización de un sistema de mesocosmos: patrones de coexistencia. Rev Colomb Cienc Pecu 2008; 21(3):480481.

8. Prieto MJ, De la Cruz L, Morales MA. Cultivo experimental del cladocero Moina sp usando como alimento Ankistrodesmus sp y Saccharomyces cereviseae. Revista MVZ Córdoba 2006b; 11(1):705-714.

9. SAS/STAT. SAS User's guide: Product documentation. 6.0 ed. Cary (NC): SAS Institute, Inc.; 2012.

10. Mojica HO, Rodríguez JA, Orozco CJ. Manual de reproducción y cultivo. El Bagre rayado (Pseudoplatystoma fasciatum) INPA (regional oriental). Villavicencio, Meta: INPA-PRONATTA; 2003.
11. Ramirez-Merlano JA, Otero-Paternina AM, Corredor Santamaría W, Medina_ Robles VM, Cruz-Casallas PE, VelascoSantamaría YM. Utilización de organismo vivos como alimentación de larvas de yaque (Leiarius marmoratus) bajo condiciones de laboratorio. Orinoquia 2010; 14(1):45-58.

12. Atencio-García V, Zaniboni-Filho E, PardoCarrasco S, Arias-Castellanos A. Influência da primeira alimentação na larvicultura e alevinagem do yamú Brycon siebenthalae. Acta Sci Anim Sci 2003; 25(1):61-72.

13. Marciales-Caro LJ, Diaz-Olarte JJ, MedinaRobles VM, Cruz-Casallas PE. Evaluación del crecimiento y sobrevivencia de larvas de bagre rayado Pseudopatystoma fasciatum (Linneaus, 1766) alimentadas con alimento vivo natural y enriquecido con ácidos grasos. Rev Colomb Cienc Pecu 2010; 23:308-316.

14. Diaz-Olarte JJ, Cruz-Casallas NE, MarcialesCaro LJ, Medina-Robles VM, Cruz-Casallas PE. Efectos de la densidad de siembra y dsiponibilidad de alimento sobre el desarrollo y sobrevivencia de las larvas de Pseudoplatystoma fasciatum. Orinoquia 2009; 13(1):21-30.

15. Nuñez J, Dugué R, Corcuy Arana N, Duponchelle F, Renno JF, Raynaud T, et al. Induced breeding and larval rearing of Surubí, Pseudoplatystoma fasciatum (Linnaeus, 1766), from the Bolivian Amazon. Aquacult Res 2008; 39(7):764-776.

16. Gaviria S, Aranguren N. Especies de vida libre de la subclase Copepoda (Arthropoda, Crustacea) en aguas continentales de Colombia. Biota Colombiana 2007; 8(1):53-68.

17. Atencio-Garcia VJ, Zaniboni-Filho E. El canibalismo en la larvicultura de peces. Rev MVZ Córdoba 2006; 11(1):9-19. 